\title{
Shigella manipulates host immune responses by delivering effector proteins with specific roles
}

\author{
Hiroshi Ashida $^{1 *}$, Hitomi Mimuro ${ }^{2}$ and Chihiro Sasakawa ${ }^{1,3,4}$ \\ ${ }^{1}$ Division of Bacterial Infection Biology, Institute of Medical Science, University of Tokyo, Tokyo, Japan, ${ }^{2}$ Division of \\ Bacteriology, Department of Infectious Diseases Control, International Research Center for Infectious Diseases, The Institute \\ of Medical Science, University of Tokyo, Tokyo, Japan, ${ }^{3}$ Nippon Institute for Biological Science, Tokyo, Japan, ${ }^{4}$ Medical \\ Mycology Research Center, Chiba University, Chiba, Japan
}

The intestinal epithelium deploys multiple defense systems against microbial infection to sense bacterial components and danger alarms, as well as to induce intracellular signal transduction cascades that trigger both the innate and the adaptive immune systems, which are pivotal for bacterial elimination. However, many enteric bacterial pathogens, including Shigella, deliver a subset of virulence proteins (effectors) via the type III secretion system (T3SS) that enable bacterial evasion from host immune systems; consequently, these pathogens are able to efficiently colonize the intestinal epithelium. In this review, we present and select recently discovered examples of interactions between Shigella and host immune responses, with particular emphasis on strategies that bacteria use to manipulate inflammatory outputs of host-cell responses such as cell death, membrane trafficking, and innate and adaptive immune responses.

Keywords: Shigella, effector, inflammation, innate immunity

\section{Introduction}

Shigella is a causative agent of bacillary dysentery, which ultimately leads to severe bloody and mucous diarrhea (shigellosis). Most cases of shigellosis occur in developing countries and affect children under 5 years old. Although antibiotics are the standard care for shigellosis patients, antibiotic-resistant bacterium is becoming common. Therefore, it is urgently necessary to develop a safe and effective Shigella vaccine. Shigella have neither adherence factor nor flagella, but they are capable of efficiently invading the intestinal epithelium. Shigella injects a subset of effectors (secreted virulence proteins) via a type III secretion system (T3SS) (protein delivery system) into host cells, allowing the bacterium to invade, multiply within the intestinal epithelium, and subvert cellular and immune functions during bacterial internalization $(1,2)$. When Shigella cells are ingested via the oral route, the bacteria move down to the colon and rectum, and then preferentially enter the $M$ cells overlying the follicle-associated epithelium of the Peyer's patches $(3,4)$ (Figure 1). Once the bacteria are endocytosed by the $\mathrm{M}$ cells, they are transcytosed toward the $\mathrm{M}$ cell pocket, where resident macrophages receive the bacteria. However, Shigella can disrupt the vacuolar membranes, disseminate into the cytoplasm, and multiply therein (5). Bacterial multiplication within the macrophages results in massive inflammatory cell death (6) (Figure 1). Meanwhile, Shigella cells that are released from dying macrophages subsequently enter the surrounding epithelium via the basolateral surface. Upon epithelial cell contact, the bacteria deliver a subset of T3SS effector proteins that trigger actin rearrangement, promoting bacterial uptake (7). Next, the Shigella cells are surrounded by a vacuolar membrane, but the bacteria rapidly disrupt this membrane and 


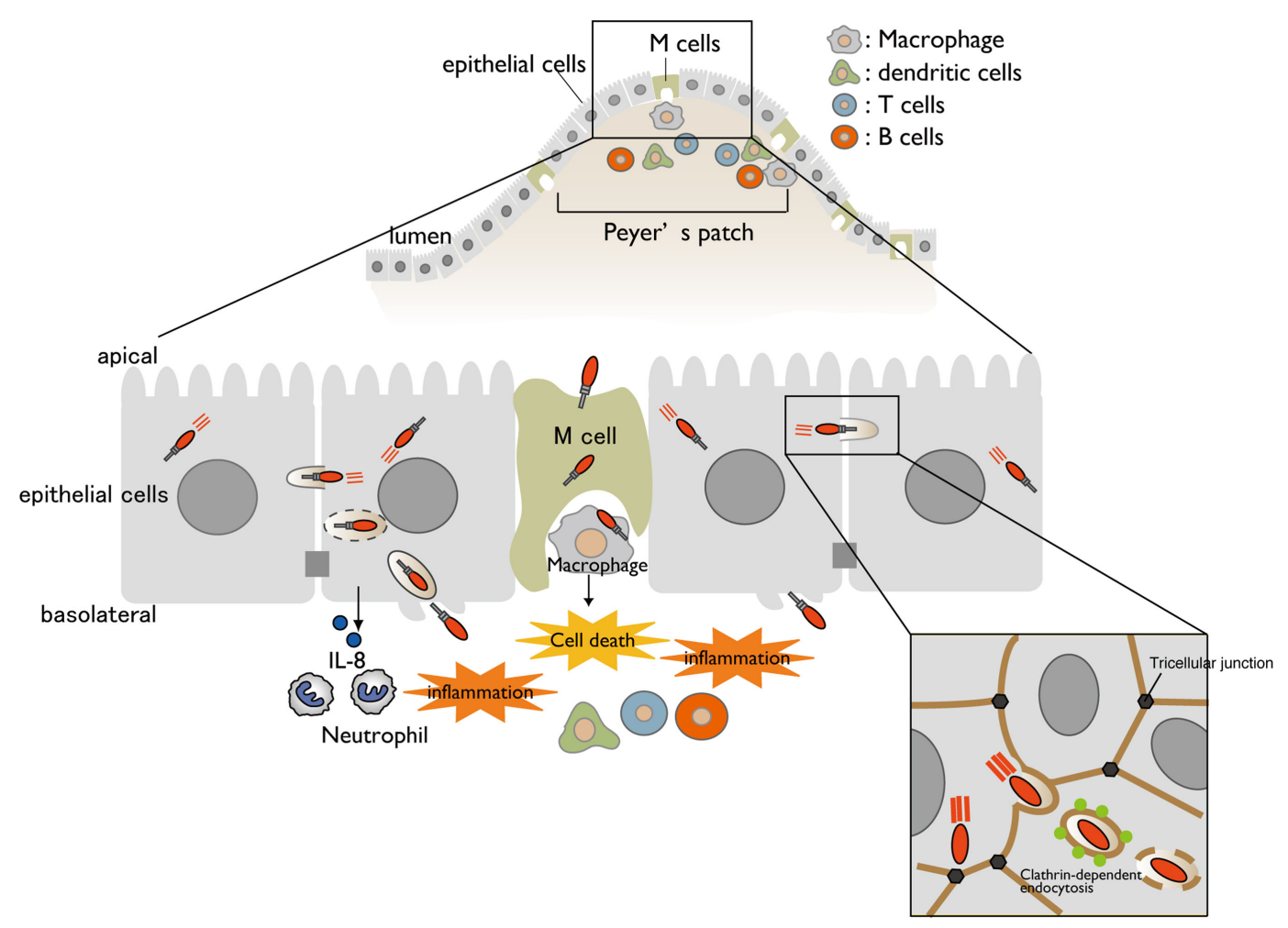

FIGURE 1 | A model for Shigella infection of the intestinal epithelium. A schematic representation of Shigella infection. Bacterial invasion and multiplication within macrophages and epithelial cells cause subsequent massive inflammatory colitis, termed as shigellosis. Cell-to-cell movement of Shigella occurs at tricellular junctions via clathrin-dependent endocytosis.

disseminate into the cytoplasm. As Shigella proliferates within the cytoplasm, it moves by inducing actin polymerization at one pole of the bacterium, providing the propulsive force required for inter- or intracellular movement (8-10). Intriguingly, Shigella cell-to-cell movement preferentially occurs at epithelial tricellular junctions, where three cells meet (Figure 1). At these positions, bacteria-containing pseudopodia are engulfed by neighboring cells via a clathrin-dependent endocytic pathway, resulting in dissemination of Shigella (11). By repeating these processes, the bacteria efficiently multiply by constantly renewing their replicative compartment. Thus, multiple infectious events during Shigella infection, including macrophage cell death, invasion of and multiplication within epithelial cells, cell-to-cell spreading, demise of the host epithelium, and alteration of the host inflammatory response, are major pathogenic events that lead to shigellosis (2) (Figure 1).

\section{Cell Death}

Host-cell death in response to microbial infection is an intrinsic immune defense stratagem against microbial intrusion. The sacrifice of infected cells plays a pivotal role in clearance of damaged cells, elimination of pathogens, local confinement of tissue damage and inflammation, and presentation of bacteriaderived antigens to the adaptive immune system (12). Cell death induced by bacterial infection can be classified into at least three types, depending on the type of cell and stage of infection: apoptosis, necrosis, and pyroptosis. Apoptosis is a non-inflammatory programed cell death triggered by the mitochondria-mediated pathway and receptor-mediated pathway, which eventually induce caspase activation (caspase-2, -3 , $-6,-7,-8,-9$, and -10 ), chromatin condensation, cell shrinkage, plasma membrane blebbing, and cytoplasm retained in apoptotic bodies. On the other hand, necrosis is an inflammatory form of cell death characterized by cell swelling, membrane rupture, and intracellular content leakage. Pyroptosis is pro-inflammatory, lytic, and programed cell death that is accompanied by activation of caspase-1 or caspase-11 (human homologs: caspase-4/5) inflammasomes, leading to the production of IL-1 $\beta$ and IL-18.

When bacteria invade and multiply within host cells, they release bacterial components [e.g., lipopolysaccharide (LPS) and peptidoglycan (PGN)] and T3SS components, and further cause infection-associated cellular damage. These are recognized as pathogen-associated molecular patterns (PAMPs) and danger-associated molecular patterns (DAMPs) by recognition receptors, such as toll-like receptors (TLRs), nucleotide-binding oligomerization domain-like receptors (NLRs), AIM2-like receptors (ALRs), and RIG-like receptors, and trigger host immune responses against bacterial infection. Upon recognition of these PAMPs and DAMPs, a subset of NLRs (e.g., NLRP1, NLRP3, and NLRC4) and ALRs (e.g., AIM2) form inflammasomes, which are multi-protein signaling complexes composed of NLR/ALR, the adaptor protein ASC, and inflammatory caspase, such as caspase1 (canonical inflammasomes) and caspase-11 (non-canonical 

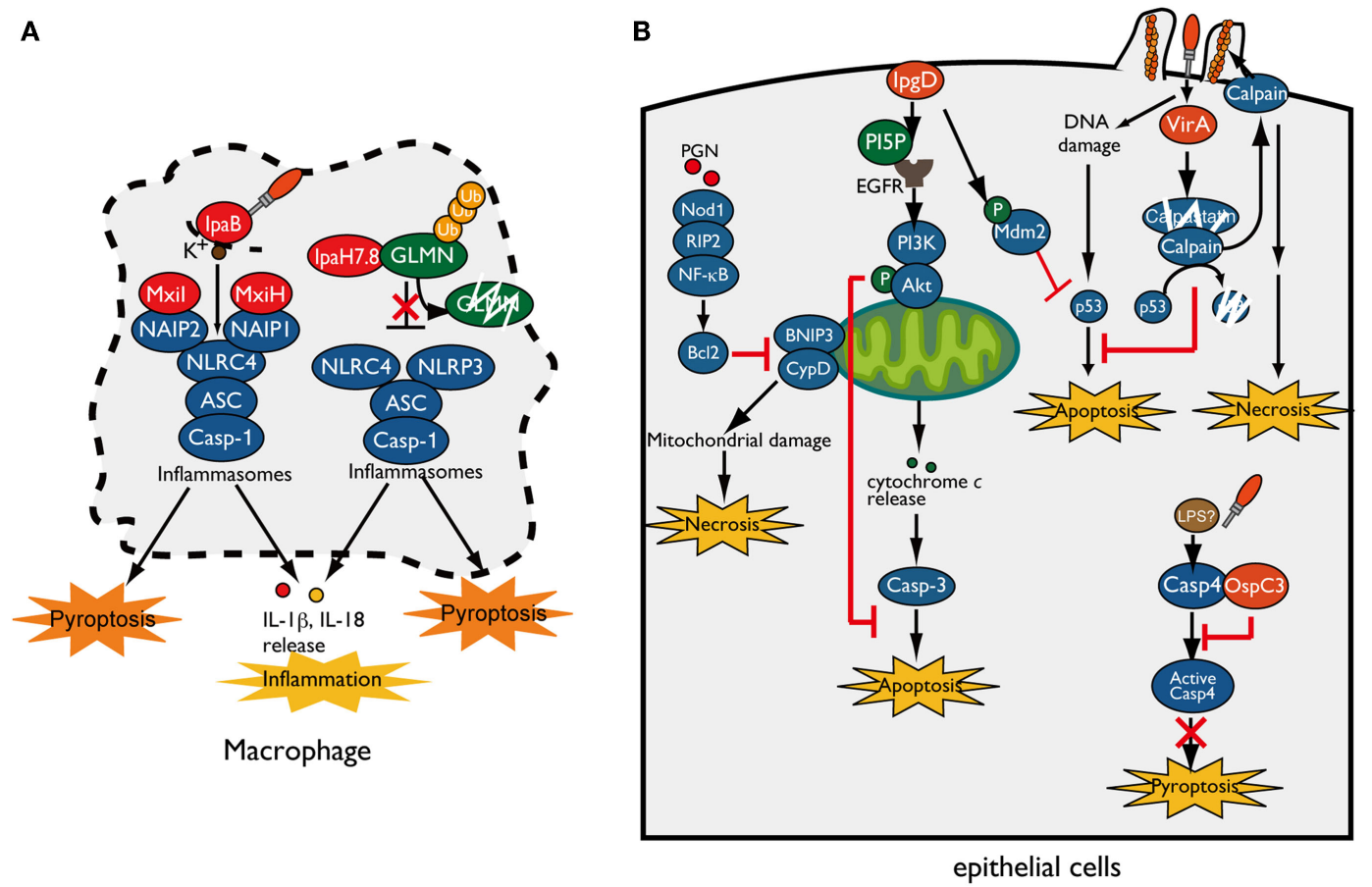

FIGURE 2 | Shigella manipulates host-cell death. (A) Shigella invasion and replication in macrophages trigger NLRC4/NLRP3-inflammasomes activation and pyroptotic cell death. The Shigella T3SS rod component Mxil and needle protein MxiH trigger NAIP2- and NAIP1-dependent NLRC4-inflammasome activation, respectively, whereas T3SS effector IpaB-mediated potassium influx triggers NLRC4-inflammasome activation and ultimately induces pyroptosis. IpaH7.8 ubiquitinates GLMN, and undergoes proteasome-dependent degradation, resulting in NLRP3/NLRC4-inflammasome activation and pyroptosis. (B) Shigella counteracts mitochondrial damage-dependent necrosis through BNIP3 and CypD by activating the PGN/Nod1/RIP2/NF- $\mathrm{KB} / \mathrm{Bcl} 2$ pro-survival pathway. Furthermore, Shigella prevents epithelial cell death by delivering an array of T3SS effectors. PI5P, generated by IpgD, promotes EGFR activation, which contributes to sustained activation of the PI3K-Akt survival pathway. IpgD and VirA target Mdm2 and calpain, respectively, to prevent p53-dependent apoptosis. OspC3 binds to the caspase-4 subunit and inhibits its activation, thereby blocking caspase-4-dependent inflammatory cell death. inflammasomes) $(13,14)$. Inflammasome activation ultimately results in release of pro-inflammatory cytokines (IL-1 $\beta$ and IL-18) and induction of pro-inflammatory lytic cell death (pyroptosis).

When Shigella invade and multiply within macrophages, they rapidly induce pyroptotic cell death accompanied by NLRP3or NLRC4-inflammasome activation, leading to IL-1 $\beta$ and IL18 secretion (15-18). T3SS needle or rod components indirectly activate NLRC4 inflammasomes through members of the NAIP subfamily of NLRs, which act as direct pathogen recognition sensors and determine the specificity of the NLRC4-inflammasome for various bacterial ligands. Recent reports have revealed that human NAIP and mouse NAIP1 bind to Shigella T3SS needle protein $\mathrm{MxiH}(19,20)$. In addition, NAIP2 binds to the T3SS inner rod component MxiI $(16,21)$ (Figure 2A). Although Shigella lacks flagella, NAIP5 and NAIP6 specifically activate the NLRC4inflammasome in response to other bacterial flagellins (22-25). Once these NAIP proteins bind to their ligands, they bind to NLRC4 and induce NLRC4-inflammasome activation and pyroptosis. In addition to bacterial T3SS components, cellular damage caused by Shigella also triggers inflammasome activation and pyroptosis. As Shigella cells multiply within macrophages, the T3SS effector IpaB assembles an ion channel within cell membrane to allow for potassium influx, which is recognized by the
NLRC4-inflammasome and ultimately triggers pyroptosis (26) (Figure 2A).

As described above, because pyroptosis is accompanied by inflammation that would limit bacterial infection, it had not been unclear whether pyroptosis is beneficial for bacterial infection (27). However, a recent study showed that induction of inflammasome activation and pyroptosis in infected macrophages is a Shigella strategy that can promote bacterial survival and dissemination. Suzuki et al. showed that Shigella induces rapid macrophage pyroptosis via IpaH7.8, an IpaH family effector, mediated NLRP3- and NLRC4-dependent inflammasome activation (28) (Figure 2A). IpaH family effectors, which have a novel E3 ubiquitin ligase activity, are widely conserved among Gram-negative bacterial pathogens, including Shigella, Salmonella, Yersinia, and Pseudomonas spp. (29). IpaH7.8 targets GLMN (glomulin/flagellar-associated protein 68), a Cullin-RING E3 ligase inhibitor, for ubiquitination and undergoes proteasomedependent degradation (Figure 2A) (28). Because GLMN acts as a negative regulator of NLR inflammasomes and pyroptosis, degradation of this factor induces inflammasome activation and pyroptosis. Consistent with results obtained in vitro, mice intranasally infected with Shigella WT or $\Delta i p a H 7.8 / W T$ complement strains induce more severe inflammatory responses and elevated numbers of colonized bacteria relative to $\Delta i p a H 7.8$ or 
$\triangle$ ipaH7.8/CA E3 ligase-deficient mutant complemented strains (28). Therefore, IpaH7.8-mediated macrophage cell death is prerequisite for allowing bacteria to escape from macrophages, further enter surrounding epithelial cells, and spread to neighboring cells.

\section{Epithelial Cell Death}

When Shigella invade and multiply within epithelial cells, the cells generate an early genotoxic stress, mitochondrial damage, oxidative stress, and recognize PAMPs and DAMPs, which could induce several types of cell death as part of the host defense system aimed at terminating bacterial infection. However, in contrast to macrophage infection, Shigella seems to prevent epithelial cell death until the bacteria have fully multiplied, because it prefers these cells as replicative niche, spread to neighboring cells, and evasion of immune cells (30) (Figure 2B). To support this notion, Shigella has several countermeasures that inhibit epithelial cell death: (i) prevention of mitochondrial damage, (ii) activation of cell survival signaling [e.g., phosphoinositide-3 kinase (PI3K)Akt and transcription factor nuclear factor $\kappa \mathrm{B}(\mathrm{NF}-\kappa \mathrm{B})]$, and (iii) prevention of caspase activation. For example, Shigella prevents necrotic cell death mediated by mitochondrial damage (through BNIP3 and CypD) by activating the Nod1-RIP2-NF- $\mathrm{KB}-\mathrm{Bcl}-2$ pro-survival pathway (31) (Figure 2B). When Shigella invades epithelial cells, it delivers the T3SS effector IpgD (a homolog of Salmonella SopB), a phosphoinositide phosphatase that converts phosphatidylinositol 4,5-bisphosphate (PIP2) into phosphatidylinositol 5-phosphate (PI5P), at the bacterial entry site (32). Because PI plays pivotal roles in actin cytoskeleton rearrangement, elevated levels of PI5P at the plasma membrane promotes bacterial invasion. Furthermore, IpgD-mediated PI5P is an important factor involved in cell survival (33). At this step, the PI5P generated by IpgD contributes to epidermal growth factor receptor (EGFR) activation, which sustains the PI3K/Akt pro-survival pathway and thereby contributes indirectly to augmentation of pro-survival signaling $(33,34)$ (Figure 2B). A recent report showed that the early stage of Shigella infection induces genotoxic stress in epithelial cells, followed by 553 pro-apoptotic signaling activation and induction of apoptosis; however, Shigella promotes p53 degradation and antagonizes the early stage of cell death by delivering two T3SS effectors, IpgD and VirA (Figure 2B) (35). As described above, IpgD promotes Akt activation, which in turn phosphorylates and stabilizes the downstream E3 ubiquitin ligase $\mathrm{Mdm} 2$. Activated Mdm2 targets p53 for ubiquitination and leads to proteasome-dependent degradation, thereby inhibiting pro-apoptosis signaling by $\mathrm{p} 53$. Furthermore, VirA promotes further degradation of $\mathrm{p} 53$ by activating calpain protease, which is also important for bacterial invasion. VirA binds to the calpain inhibitor calpastatin and promotes its degradation, resulting in degradation of p53 by activated calpain and blocking the p53 pro-apoptotic signaling pathway. Although VirA-mediated calpain activation promotes bacterial entry and prevents the early stage of apoptosis, sustained calpain activation ultimately induces necrosis and restricts bacterial proliferation (35). In addition, Shigella exploits calpain activation and mitochondrial function to suppress the innate immune response without inducing host-cell death (see below) (36). These results indicate that Shigella deploys a sophisticated strategy that controls the delicate balance of hostcell death until Shigella has succeeded in primary colonization and proliferation within epithelial cells.

Recent reports have shown that non-canonical caspase-4/-11 inflammasome activation is an essential host defense mechanism of epithelial cells against enteric bacterial pathogens such as Shigella, Salmonella, and enteropathogenic Escherichia coli (EPEC). Caspase-4/-11 directly binds to cytoplasmic LPS of Gram-negative bacterial pathogens and activates caspase-4/-11mediated inflammasomes and pyroptosis, ultimately inducing epithelial cell shedding to eliminate infected cells (37-39). However, Shigella antagonizes caspase-4-dependent inflammatory cell death by delivering the T3SS effector OspC3 and promoting epithelial infection (37). OspC3 deploys a unique mechanism that specifically targets and inactivates caspase-4, but not caspase1 or mouse caspase-11. The C-terminal ankyrin-repeat (ANK) region of OspC 3 interacts with the p19 subunit of caspase- 4 and prevents its activation by inhibiting p19 and p10 dimerization (Figure 2B). In human epithelial cell lines, the Shigella $\Delta$ ospC3 mutant induces early caspase-4-dependent pyroptotic cell death and increased cytokine production relative to that of WT Shigella. Of note, the Shigella $\Delta$ ospC3 mutant also exhibited severe mucosal cell death and a reduction in the number of colonizing bacteria in a guinea pig rectal infection model, indicating the importance of OspC3-mediated cell death inhibition for bacterial infection (37). Thus, Shigella delivers a subset of T3SS effector proteins that delay epithelial cell death until the bacterial cells have fully replicated and further disseminated into surrounding cells.

\section{Autophagy}

Autophagy is an essential cellular catabolic process, which targets proteins, organelles, and large protein aggregates by sequestering deleterious cargos within a double-membrane compartment, the autophagosome. Autophagy also plays a pivotal role as a part of the innate immune system, by acting as a cytosolic sensor to recognize DAMPs and PAMPs, and as an "executioner" that engulfs bacteria in autophagosomes that fuse with lysosomes, ultimately destroying bacteria within lysosomal compartments.

Shigella enter epithelial cells, disseminate into the cytosol by disrupting the surrounding phagosomal membrane, and move into adjacent cells by inducing actin polymerization at one bacterial pole. In Shigella invasion of epithelial cells, Nod1 and Atg16L1 are recruited to the plasma membrane beneath the Shigella entry site and subsequently trigger autophagy (40). Furthermore, hostcell vacuolar membrane remnants generated by Shigella are recognized as DAMPs by galectin-8, and these remnants are also polyubiquitinated, followed by the recruitment of p62 (ubiquitin adaptor protein) and LC3 (autophagosome marker), resulting in autophagic activation $(41,42)$ (Figure 3). During multiplication within the cytosol, Shigella outer membrane protein VirG (IcsA) accumulates at one pole of the bacterial surface. There, VirG recruits and activates N-WASP, which subsequently recruits and activates the Arp $2 / 3$ complex, thereby inducing actin polymerization and bacterial motility within the cell (8-10). At this stage, VirG is recognized by the host autophagy protein Atg5, a protein essential for autophagosome maturation, resulting in Shigella uptake by autophagosomes. However, Shigella prevents 


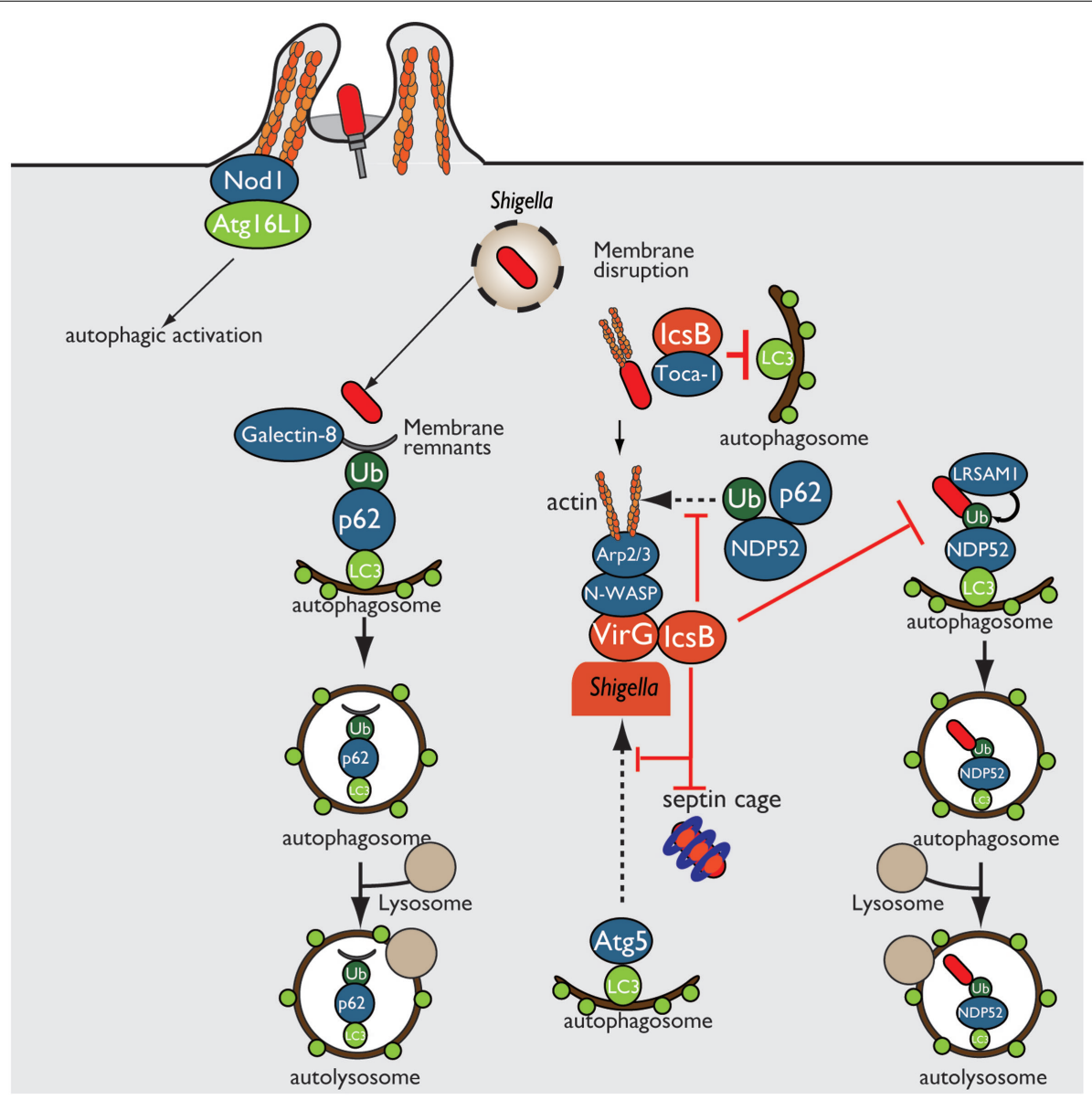

FIGURE 3 | Shigella prevents autophagic recognition. Shigella prevents autophagic clearance by evading autophagic recognition. Shigella delivers the T3SS effector IcsB, which binds to the bacterial outer membrane protein VirG and prevents Atg5-VirG interaction and ubiquitin recruitment, thereby evading autophagic recognition. IcsB also recruits Toca-1, and prevents the recruitment of LC3 to around intracellular bacteria. autophagic recognition by delivering the IcsB T3SS effector. At an early stage of infection, IcsB binds to and recruits Toca-1, which is required for efficient formation of actin polymerization, around intracellular bacteria, and IcsB-Toca-1 prevents the recruitment of LC3 $(43,44)$. Furthermore, at the later stage of infection, IcsB plays a pivotal role in camouflage against autophagic recognition (45). IcsB and Atg5 interact with the same region on VirG, but the affinity of IcsB for VirG is stronger than that of Atg5; therefore, IcsB competitively inhibits the VirG-Atg5 interaction, masking the target VirG protein from autophagic recognition (45) (Figure 3). Consistent with this, the Shigella $\Delta i c s B$ mutant is trapped in the autophagosome and delivered for lysosomal degradation (45). Intriguingly, IcsB has cholesterol binding region, and its ability is involved in evading autophagic recognition without affecting IcsB-VirG binding (46). In Shigella infection, ubiquitindependent selective autophagy is also triggered, but the bacteria evade ubiquitin recognition. Although Shigella WT is not targeted by ubiquitin, LRSAM1 (a mammalian LRR-containing RING E3 ligase), which itself acts as a bacterial recognition molecule, localizes to the Shigella $\Delta i c s B$ mutant, and LRSAM1-mediated bacterial ubiquitylation has been observed in vitro (44). LRSAM1 recognition can trigger ubiquitin-dependent selective autophagy, resulting in restriction of bacterial replication (47). In addition, another host factor septin, which is GTP-binding protein, assembles at sites of VirG-induced actin polymerization and forms cages that surrounding bacteria, and prevents inter- and intracellular movement, thereby targeting by autophagy and restricting bacterial proliferation (48). A recent report showed that the ubiquitin adaptors p62 and NDP52 target Shigella for autophagy in an actin polymerization- and septin-dependent manner, and that the Shigella $\Delta$ virG mutant reduces the recruitment of p62 and NDP52 (49). The Shigella $\Delta i c s B$ mutant increases septin-cage formation and the recruitment of ubiquitin, p62, and NDP52. Therefore, although VirG-mediated actin polymerization is targeted by septin- and ubiquitin-dependent selective autophagy, the IcsB-VirG interaction prevents the recruitment of ubiquitin, p62, and NDP52 around bacteria (49) (Figure 3).

\section{Membrane Trafficking}

Intracellular trafficking of membranes and proteins is essential for maintenance of epithelial homeostasis and barrier function, and also acts as a host defense system against bacterial pathogens. In eukaryotic cells, intracellular trafficking system can be divided 
into two pathways, endocytosis and the secretory pathway. During endocytosis, which internalizes extracellular molecules or delivers plasma membrane proteins to specialized sites, the cargo is sorted in the early endosome and further transported to Golgi, endoplasmic reticulum (ER), or late endosomes and lysosome for degradation, whereas some cargo proteins are transported back to the plasma membrane via recycling endosomes. On the other hand, the secretory pathway, which produces molecules such as antimicrobial peptides, growth factors, cell surface receptors, and cytokine secretion, transport molecules from the ER to the plasma membrane through the Golgi.

Exocytosis, endocytosis, phagocytosis, and cytokine secretion play essential roles as host defense systems for eliminating invading bacterial pathogens. However, many intracellular bacterial pathogens, such as Shigella, Salmonella, and Legionella, hijack and exploit host intracellular trafficking system to enter host cells, evade subsequent phagocytic destruction, and establish their safety replicative niche for their survival and proliferation $(7,50$, 51). Shigella enter non-phagocytic cells by delivering a coordinated set of T3SS effector proteins that trigger actin cytoskeleton or plasma membrane remodeling, and promote subsequent bacterial uptake by host cells. Soon after internalization, the bacteria are engulfed within a membrane-bound vacuole that is derived from the host plasma membrane. After that, Shigella disrupt and escape from this vacuole and replicate within the cytoplasm (7). Vacuolar disruption or modulation by intracellular bacteria is related to the host membrane trafficking system, which is tightly regulated by small GTPases of the Rab and ARF families. The small GTPases act as molecular switches that cycle between the GTP-bound active form and GDP-bound inactive form, catalyzed by two classes of regulatory proteins, guanine nucleotide exchange factors (GEFs) and GTPase-activating proteins (GAPs). GEFs exchange GDP for GTP to activate GTPases, whereas GAPs inactivate GTPases by promoting hydrolysis of GTP to GDP (52). Rab and ARF GTPases play an important role in resisting bacterial infection, such as secretion of cytokines and antimicrobial peptides, maintenance of epithelial barrier integrity, and maturation of phagosomes into lysosomes to degrade entrapped bacteria. Therefore, Shigella target Rab GTPases and interfere with host membrane trafficking by delivering T3SS effector proteins (Figure 4).

In Shigella infection, the endocytic pathway is essential event for bacterial invasion, vacuolar rupture, and spread to neighboring cells. Using a high-content siRNA screening, Mellouk et al. recently demonstrated that Shigella targets and recruits Rab11, a component of host recycling endosomes, to disrupt the vacuole and efficiently escape from it by delivering the IpgD effector (53) (Figure 4). As described before, IpgD is a phosphoinositide phosphatase that produces PI5P, which is important for vesicular trafficking. In cells infected with Shigella $\Delta i p g D$ or phosphataseinactive $\Delta i p g D / i p g D$ C438S complemented mutant, Rab11 is not recruited to the site of bacterial invasion, and vacuolar rupture is delayed; however, this deficiency is rescued by the complementation of $\Delta i p g D / i p g D$ WT or WT Shigella infection, highlighting the requirement for the phosphoinositide phosphatase activity of IpgD. Furthermore, vacuole disruption by Shigella is delayed in Rab11 knockdown epithelial cells without affecting bacterial

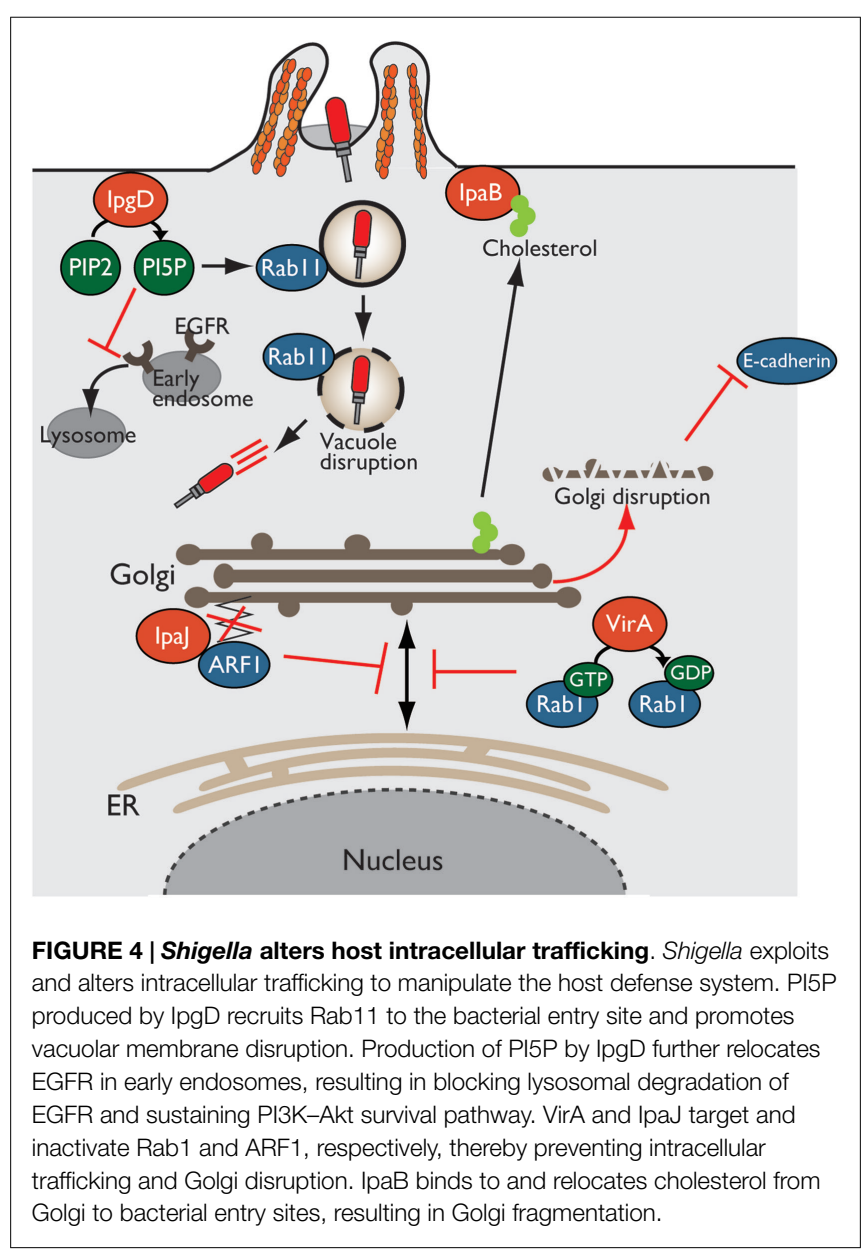

invasion (53). Therefore, Shigella targets Rab11 to disrupt the vacuole and allow bacterial escape into the cytoplasm and promote further bacterial replication and dissemination.

In addition, as mentioned above briefly, IpgD subverts host intracellular trafficking to promote host-cell survival for bacterial prolonged colonization (34). The production of PI5P by IpgD at bacterial entry sites recruits active EGFR, which is required for the PI3K-Akt survival signaling pathway. An elevated level of PI5P at the plasma membrane, due to the activity of IpgD, alters EGFR trafficking and relocates it to early endosomes from late endosomes or lysosomes, thereby blocking lysosomal degradation of EGFR and sustaining EGFR-PI3K-Akt survival signaling activation (34) (Figure 4).

The Golgi apparatus is a central organelle involved in protein or lipid transport in eukaryotic cells. The Golgi processes and sorts proteins made by the ER, and transport them to various destinations, such as the plasma membrane and lysosome. Therefore, defects in Golgi function result in the loss of intracellular trafficking, including secretion of antimicrobial peptides and cytokines, and transport of epithelial junction proteins that are essential for epithelial barrier function against bacterial infection. Intriguingly, Shigella disrupts the Golgi apparatus by inhibiting vesicle trafficking in infected epithelial cells in a T3SS-dependent manner. Of note, three Shigella effectors, VirA, IpaJ, and IpaB, play 
important roles in disrupting the Golgi apparatus by targeting host intracellular trafficking (Figure 4).

Recent reports have shown that VirA and IpaJ, which have specific enzymatic activities targeting Rab and ARF GTPases, respectively, dampen host antibacterial defense systems by inhibiting the host secretory pathway. VirA (a homolog of EPEC EspG), which has TBC (homologous catalytic domain of GAP for Rab GTPase)-like GAP activity, preferentially targets and catalyzes GTP hydrolysis in Rab1, which localizes in the ER and mediates ER-to-Golgi trafficking (54). VirA inactivates Rab1, inhibits ERto-Golgi transport of Rab1-containing vesicles, and disrupts the Golgi apparatus in host epithelial cells (Figure 4). Because Rab1 is important for autophagosome formation, Rab1 inactivation by VirA counteracts antibacterial autophagy. Of note, infection by a Shigella $\Delta$ virA mutant or GAP-inactive $\Delta v$ irA/virA-RQ complemented mutant increases autophagosome formation and reduces the number of colonizing bacteria relative to that of WT Shigella in human epithelial cell lines. Thus, TBC-like GAP activity of VirA contributes to bacterial escape from autophagy and intracellular survival by blocking intracellular trafficking through Rab1 GTPase inactivation (54).

A bioinformatics approach revealed that IpaJ belongs to the cysteine protease family, whose members have Cys-His-Asp catalytic triad residues $(55,56)$. Further yeast genetic screening and mass spectrometry analysis demonstrated that IpaJ specifically cleaves the $N$-myristoylated glycine from ARF1 (Figure 4). $\mathrm{N}$-myristoylation is an essential fatty-acid modification that is involved in protein localization, signal transduction, autophagosome maturation, and organelle function. Because $N$ myristoylation of ARF1 is essential for binding to the Golgi membrane, IpaJ-mediated cleavage induces the release of ARF1 from Golgi and its disruption, thereby inhibiting intracellular trafficking, including cytokine secretion and signal transduction $(55,56)$.

Another effector, IpaB, also disrupts the Golgi apparatus by targeting lipid trafficking, and thereby reduces epithelial barrier function. IpaB binds to and redirects cholesterol at the bacterial entry site from the Golgi apparatus, which depletes the lipid of Golgi and induces its fragmentation (Figure 4). The Golgi apparatus fragmentation further induces tubulation of the Rab11positive compartment and reorganizes the recycling endosome, which is involved in trafficking of E-cadherin to adherence junction, resulting in epithelial junction disruption (57).

As described above, membrane and protein trafficking, such as the phagosome component, epithelial barrier components, antimicrobial peptides, cytokines, and cell surface receptors, is a major host defense system against bacterial infection. Therefore, Shigella blocks and alters host intracellular trafficking by delivering subsets of effectors that promote infection.

\section{Manipulation of Host Innate Immunity}

Once Shigella invades and replicates within host cells, the innate immune system quickly senses PAMPs or DAMPs, and transmits various alarm signals to the rest of the immune system, and ultimately triggers inflammation. Inflammation, which is accompanied by inflammatory cytokine secretion, neutrophil recruitment, and massive tissue destruction, is the hallmark of the host innate immune response that eventually restricts and eliminates bacterial infection. However, many bacterial pathogens, including Shigella, deliver a subset of T3SS effectors that manipulate host innate immune responses, thereby promoting bacterial colonization and survival (2) (Figure 5).

\section{ATP Release}

During bacterial infection, various molecules are released from damaged or stressed cells as DAMPs (e.g., ATP, uric acid, and membrane remnants), which trigger inflammatory immune responses. For example, infection of intestinal epithelial cells with enteric bacterial pathogens, such as Shigella, Salmonella, and EPEC, induce connexin hemichannel-dependent ATP release, which acts as an endogenous danger alarm against bacterial infection and triggers inflammatory responses (58). Hemichanneldependent ATP release is a common host defense system against enteric bacterial infection. To counteract ATP-dependent inflammation, Shigella blocks ATP release by delivering IpgD to epithelial cells. IpgD blocks hemichannels and prevents ATP release through production of PI5P, which regulates hemichannel opening, thereby dampening ATP-dependent inflammation (Figure 5). Consistent with this, in both in vitro and in vivo infections, the Shigella $\Delta i p g D$ mutant causes elevated ATP release, severe intestinal inflammation, and mucosal damage, illustrating the key role of IpgD in preventing ATP-dependent inflammation (58).

\section{Manipulation of NF- $\kappa B$ Signaling}

During bacterial infection, the transcription factor NF- $\kappa B$, the master regulator of pro-inflammatory cytokines, plays a major role in mediating an inflammatory signaling pathway that triggers a wide range of host inflammatory responses. In response to bacterial infection, several intracellular and extracellular stimuli activate signal transduction cascades, and NF- $\mathrm{KB}$ is translocated into the nucleus, where it promotes the transcription of target genes (59). In particular, ubiquitination of signaling factors is prerequisite for regulation of NF- $\kappa \mathrm{B}$ activity; therefore, many bacterial pathogens, including Shigella, inhibit NF- $\kappa \mathrm{B}$ activation by targeting signaling factors and altering signal transduction, thereby dampening inflammation, in order to promote infection (60).

Invasion of epithelial cells by Shigella produces membrane ruffles by remodeling the actin cytoskeleton around the bacterial entry site. Aberrant membrane ruffles, which protrude from the bacterial entry site and are accompanied by diacylglycerol (DAG) production, are sensed as DAMPs by the host innate immune system and trigger the activation of the DAGCBM (CARMA-BCL10-MALT1)-TRAF6-NF- $\mathrm{BB}$ pathway. However, Shigella delivers OspI via the T3SS into the host cells; this factor targets and deamidates UBC13 (converts Gln-100 to Glu-100), an E2 required for TRAF6 E3 activity, resulting in abolition of its E2 activity and thereby interfering with the DAG-CBM-TRAF6-NF- $\kappa B$ pathway (61) (Figure 5).

Following membrane ruffling, Shigella-mediated vacuolar membrane ruptures are also recognized as DAMPs, triggering an additional alarm-signaling pathway via recruitment and activation of $\mathrm{PKC}$, ultimately leading to the activation of the PKC-NF- $\kappa \mathrm{B}$ pathway. To counteract PKC-NF- $\kappa \mathrm{B}$ activation, 


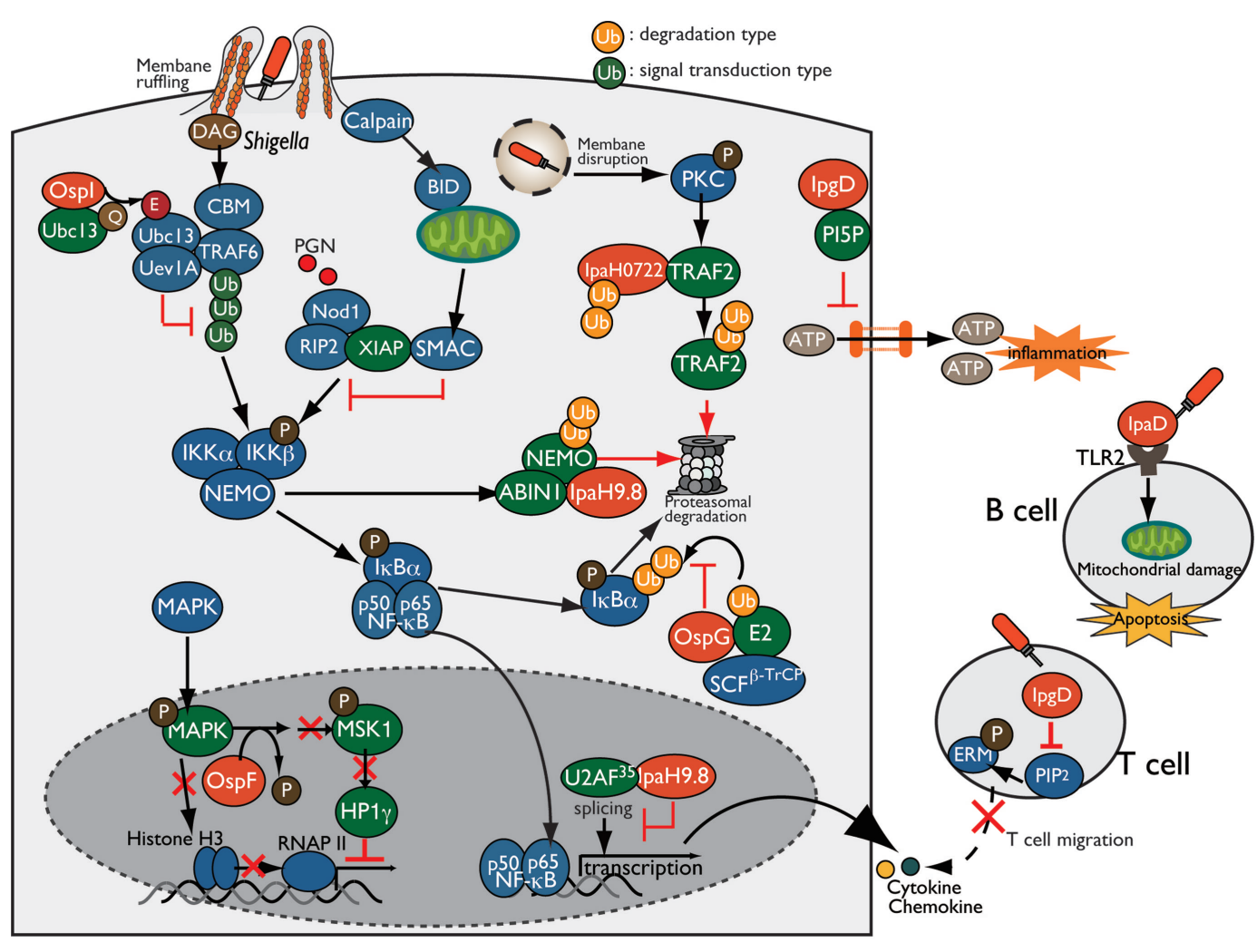

FIGURE 5 | Shigella manipulates the host innate and adaptive immune response. Shigella manipulates host inflammatory responses by delivering a subset of T3SS effectors, including IpgD, Ospl, OspG, OspF, and IpaH. PI5P, generated by IpgD, prevents ATP release-dependent inflammation through hemichannels. OspG binds to ubiquitin and ubiquitinated E2 proteins and prevents $1 \kappa B \alpha$ ubiquitylation, which is required for NF- $\kappa B$ activation. Ospl deamidates and inactivates Ubc13, resulting in inhibition of TRAF6 ubiquitination. IpaH0722 and IpaH9.8 target TRAF2 and NEMO, respectively, for ubiquitination, and undergo proteasome degradation. In addition, IpaH9.8 further targets U2AF35, preventing its participation in the splicing reaction via an unidentified bacterial factor, Shigella induces calpain-dependent BID activation, which, in turn, releases mitochondrial SMAC to antagonize XIAP-mediated inflammation. IpgD-mediated hydrolysis of PIP2 inactivates ERMs of T cells, which crosslink actin filaments with the plasma membrane, thereby preventing $T$ cell migration. Shigella induces B cell death in both invaded and non-invaded cells. In non-invaded cells, the T3SS needle-tip protein IpaD interacts with TLR2 on $\mathrm{B}$ cells and triggers mitochondrial damage, which eventually induces apoptosis.
Shigella delivers IpaH0722, one of the IpaH family of E3 ubiquitin ligase effectors. IpaH0722 dampens the acute inflammatory response by ubiquitination of TRAF2, a molecule downstream of PKC, thereby preferentially inhibiting PKC-mediated NF- $\kappa \mathrm{B}$ activation (62) (Figure 5).

During Shigella invasion of epithelial cells, Nod1 recognizes PGNs released from Shigella as PAMPs, triggering the Nod1-RIP2 pathway and activating the downstream mitogen-activated protein kinase (MAPK) and NF- $\mathrm{KB}$ signaling pathways. Shigella delivers IpaH9.8, an IpaH family E3 ubiquitin ligase effector, which preferentially prevents the Nod1-dependent NF- $\kappa \mathrm{B}$ activation. IpaH9.8 interacts with NEMO/IKK $\gamma$, an essential component of the IKK kinase complex, and targets NEMO for ubiquitination. IpaH9.8 also interacts with ABIN-1, an ubiquitin-binding adaptor protein, to further promote polyubiquitylation of NEMO. Ubiquitinated NEMO by IpaH9.8 subsequently leads to proteasomal degradation, thereby diminishing NF- $\mathrm{KB}$ activation (63) (Figure 5). The activity of this E3 ligase effector during Shigella infection contributes to bacterial colonization in a mouse lung infection models (63).
Upon Nod1 activation, the X-linked inhibitor of apoptosis protein (XIAP) plays a crucial role in activating Nod1-dependent inflammatory responses. XIAP interacts with RIP2 and facilitates NF- $\kappa \mathrm{B}$ activation, ultimately leading to pro-inflammatory gene transcription. A recent report showed that Shigella evades XIAP-mediated inflammation by actively releasing mitochondrial SMAC (36). Shigella infection triggers calpain activation and processes and activates $\mathrm{BH} 3$-only protein $\mathrm{BID}$, which then translocates to the mitochondria and induces the release of SMAC. Released SMAC binds to XIAP, and inhibits XIAP-mediated NF$\kappa \mathrm{B}$ activation without inducing mitochondrial damage or cell death (Figure 5). Although it remains unknown whether BIDmediated release of SMAC by Shigella depends on a T3SS effector, Shigella uniquely exploits mitochondrial function to block the host innate immune response while avoiding cell death (36).

In addition, Shigella delivers another effector, OspG, which shares sequence similarity with mammalian serine/threonine kinases and inhibits NF- $\kappa B$ activation (64). OspG binds to ubiquitin and ubiquitinated E2s, which are required for phospho-IкB $\alpha$ ubiquitination by an E3 ligase such as $\mathrm{SCF}^{\beta-\operatorname{TrCP}}$, and formation 
of this OspG-E2-ubiquitin complex promotes OspG kinase activity and increases the prevention of NF- $\mathrm{KB}$ activation (64-67) (Figure 5).

\section{Epigenetic Regulation of Immune Gene Expression}

Because the host transcriptional program plays a pivotal role in triggering inflammation and eliminating bacterial infection, Shigella overcomes this defense mechanism by delivering a subset of T3SS effector proteins that regulate gene expression and counteract host immune responses. In addition to interfering with NF- $\kappa \mathrm{B}$ signaling, as described above, Shigella hacks the host immune responses by reprograming the host epigenome to promote infection. The T3SS effector OspF (homolog of Salmonella SpvC and Pseudomonas syringae HopAl1), which has a unique phosphothreonine lyase activity, translocates into the nucleus of epithelial cells, where it irreversibly dephosphorylates and inactivates MAPKs (Erk and p38) through beta-elimination of the phosphate group $(68,69)$. Inactivation of MAPK by OspF further blocks downstream phosphorylation of histone $\mathrm{H} 3$ at Ser10 at the promoters of a subset of innate immune genes, such as IL8 , and promotes chromatin condensation, resulting in repression of transcription by masking NF- $\mathrm{\kappa B}$ binding sites (68) (Figure 5). In addition to histone modification, OspF also alters the activity of the chromatin reader heterochromatin protein 1 (HP1) and represses host gene expression during Shigella infection (70). Although phosphorylated HP1 $\gamma$ at Ser83 proteins positively regulate euchromatin (a transcriptionally active state) and activate transcription, OspF inactivates Erk and consequently reduces the activity of the downstream kinase MSK1, a kinase for HP1 $\gamma$ at Ser83. As a result of HP1 $\gamma$ dephosphorylation, HP1 $\gamma$ dissociates from sites of transcriptional activation at OspF-target genes such as IL-8 (Figure 5). Consistent with this, the Shigella $\Delta o s p F$ mutant increased the level of HP1 $\gamma S 83$ phosphorylation relative to WT Shigella-infected cells (70). Therefore, OspF manipulates host transcriptional response via two epigenetic modifications: (i) decreasing the level of phosphorylated histone $\mathrm{H} 3$, and (ii) altering the activity of HP1 through dephosphorylation of MAPK, which contributes to downregulation of host inflammatory responses.

In addition to OspF, Shigella delivers IpaH9.8 to regulate proinflammatory gene expression. IpaH9.8 is translocated into the nucleus of epithelial cells, where it binds to U2AF35, an mRNA splicing factor, and inhibits the U2AF35-dependent splicing reaction, enabling the bacterium to dampen expression of numerous genes, including some that encode pro-inflammatory cytokines and chemokines (71) (Figure 5).

\section{Manipulation of Host Adaptive Immunity}

Manipulation of the host innate immune response by Shigella is a pivotal survival strategy for promoting infection; however, the interactions between Shigella and adaptive immune response, such as $\mathrm{T}$ and $\mathrm{B}$ lymphocytes, have not been thoroughly investigated. One prominent issue is the lack of appropriate animal infection models that mimic human intestinal infection. Until now, however, several studies have proven the importance of adaptive immunity against Shigella infection using a mouse pulmonary infection model that mimics the acute inflammation that occurs during shigellosis. Mice that are genetically deficient in $\mathrm{B}, \mathrm{T}$, and NK cells are much more susceptible to Shigella infection than WT mice, and T and NK cells play critical roles in clearing Shigella (72). Additional studies have shown that $\mathrm{CD}^{+}{ }^{+} \mathrm{T}$ helper 17 (Th 17) cells, which are predominantly primed in response to Shigella, produce IL-17A and eventually restrict secondary Shigella infection, indicating the involvement of Th17 cells in adaptive immunity against Shigella infection (73). By contrast, antigenspecific $\mathrm{CD}^{+} \mathrm{T}$ cells, which are usually required for adaptive immunity against cytosolic bacterial infection, are not primed and involved in adaptive immunity against Shigella infection (74). As the precise role and the importance of adaptive immunity against Shigella infection have been revealed, several recent studies have shown that host adaptive immunity is targeted and subverted by Shigella T3SS effectors (Figure 5).

$\mathrm{T}$ cell migration and activation are key events in the induction of antibody- and cell-mediated immune responses against bacterial infection. Recently, the Phalipon group investigated the interaction between $\mathrm{T}$ cells and Shigella using in vitro and in vivo approaches, and demonstrated that Shigella interferes with adaptive immune responses by targeting $\mathrm{T}$ cells. They also found that Shigella invades activated $\mathrm{CD} 4^{+} \mathrm{T}$ cells and inhibit chemoattractant-mediated $\mathrm{T}$ cell migration by delivering IpgD (75). T-lymphocyte migration toward a chemoattractant depends on the membrane cytoskeleton crosslinkers proteins ERM (ezrin, radixin, and moesin), which are tightly converted between the active and inactive conformation by the concentration of PIP2 at the plasma membrane. Because IpgD is phosphoinositide phosphatase, it subsequently hydrolyzes and decreases the concentration of PIP2 at the plasma membrane, thereby inactivating ERM (Figure 5). In addition, Shigella impairs $\mathrm{CD}^{+} \mathrm{T}$ cell dynamics within lymph nodes, where adaptive immunity is initiated, in the mouse infection model (76). Therefore, Shigella targets T-lymphocytes and inhibits their migration by delivering the T3S effector IpgD, thereby interfering with adaptive immunity.

B lymphocytes are key players in antibody-mediated immunity, and they produce and secrete cytokines that contribute to the antibody-independent immune response against bacterial infection. However, massive T- and B-cell deaths are observed in rectal biopsies of Shigella-infected humans, indicating that Shigella impairs B cell-mediated immunity (77). To support this notion, a recent report showed that Shigella targets B cells and induces cell death in both Shigella-invaded and non-invaded cells (78). Shigella invades B cells and replicates intracellularly, resulting in B cell death. Furthermore, Shigella induces B cell apoptosis via the T3SS needle-tip protein IpaD. IpaD binds to TLR2 on B cells and triggers the loss of mitochondrial dysfunction and apoptotic cell death signaling in non-invaded B cells, eventually helping the bacterium to avoid antibody-mediated immune responses (78) (Figure 5). Therefore, Shigella targets $\mathrm{T}$ and B cells and manipulates adaptive immunity against Shigella infection, thereby preventing antibody-mediated lasting immunity and promoting bacterial infection. 


\section{Conclusion}

Although bacterial infection elicits host defense systems that would restrict and eliminate bacteria, many bacterial pathogens have evolved excellent strategies to manipulate host immune responses and enable bacterial colonization. Here, we review the current understanding of how Shigella manipulates host immune responses, focusing specifically on T3SS effectormediated interference with host signaling transduction cascades, alteration of membrane trafficking, and modulation of host-cell death. Our understanding of the molecular basis of the interaction between bacterial effectors and host immune systems has advanced greatly during the past decade. Although accumulating evidence has revealed the importance of manipulation of the host immune system during bacterial infection, our knowledge of bacterial strategy is still in its infancy. Although approximately 50 T3SS effectors of Shigella are currently recognized, we have only elucidated the molecular function of one-third of them.

\section{References}

1. Parsot C. Shigella type III secretion effectors: how, where, when, for what purposes? Curr Opin Microbiol (2009) 12:110-6. doi:10.1016/j.mib.2008.12.002

2. Ashida H, Ogawa M, Mimuro H, Kobayashi T, Sanada T, Sasakawa C. Shigella are versatile mucosal pathogens that circumvent the host innate immune system. Curr Opin Immunol (2011) 23:448-55. doi:10.1016/j.coi.2011.06.001

3. Wassef JS, Keren DF, Mailloux JL. Role of M cells in initial antigen uptake and in ulcer formation in the rabbit intestinal loop model of shigellosis. Infect Immun (1989) 57:858-63.

4. Perdomo OJ, Cavaillon JM, Huerre M, Ohayon H, Gounon P, Sansonetti PJ. Acute inflammation causes epithelial invasion and mucosal destruction in experimental shigellosis. J Exp Med (1994) 180:1307-19. doi:10.1084/jem.180. 4.1307

5. High N, Mounier J, Prévost MC, Sansonetti PJ. IpaB of Shigella flexneri causes entry into epithelial cells and escape from the phagocytic vacuole. EMBO J (1992) 11:1991-9.

6. Zychlinsky A, Prevost MC, Sansonetti PJ. Shigella flexneri induces apoptosis in infected macrophages. Nature (1992) 358:167-9. doi:10.1038/358167a0

7. Carayol N, Tran Van Nhieu G. Tips and tricks about Shigella invasion of epithelial cells. Current Opin Microbiol (2013) 16:1-6. doi:10.1016/j.mib.2012. 11.010

8. Suzuki T, Miki H, Takenawa T, Sasakawa C. Neural Wiskott-Aldrich syndrome protein is implicated in the actin-based motility of Shigella flexneri. EMBO J (1998) 17:2767-76. doi:10.1093/emboj/17.10.2767

9. Suzuki T, Mimuro H, Miki H, Takenawa T, Sasaki T, Nakanishi H, et al. Rho family GTPase Cdc42 is essential for the actin-based motility of Shigella in mammalian cells. J Exp Med (2000) 191:1905-20. doi:10.1084/jem.191.11.1905

10. Egile C, Loisel TP, Laurent V, Li R, Pantaloni D, Sansonetti PJ, et al. Activation of the CDC42 effector N-WASP by the Shigella flexneri IcsA protein promotes actin nucleation by Arp2/3 complex and bacterial actin-based motility. J Cell Biol (1999) 146:1319-32. doi:10.1083/jcb.146.6.1319

11. Fukumatsu M, Ogawa M, Arakawa S, Suzuki M, Nakayama K, Shimizu S, et al. Shigella targets epithelial tricellular junctions and uses a noncanonical clathrindependent endocytic pathway to spread between cells. Cell Host Microbe (2012) 11:325-36. doi:10.1016/j.chom.2012.03.001

12. Ashida H, Mimuro H, Ogawa M, Kobayashi T, Sanada T, Kim M, et al. Cell death and infection: a double-edged sword for host and pathogen survival. J Cell Biol (2011) 195:931-42. doi:10.1083/jcb.201108081

13. Kayagaki N, Warming S, Lamkanfi M, Vande Walle L, Louie S, Dong J, et al. Non-canonical inflammasome activation targets caspase-11. Nature (2011) 479:117-21. doi:10.1038/nature10558

14. Kayagaki N, Wong MT, Stowe IB, Ramani SR, Gonzalez LC, AkashiTakamura $\mathrm{S}$, et al. Noncanonical inflammasome activation by intracellular
As has been shown for other bacterial pathogens, the effector activity and strategies described above are not unique to Shigella, but are instead shared as common strategies by many bacterial pathogens. Therefore, the discovery of new Shigella strategies that manipulate the host immune system will not only provide a new understanding of how bacteria subvert the host immune system but also facilitate development of new types of antibacterial drugs that target bacterial effectors and bacterial live vaccines to overcome bacterial infections.

\section{Acknowledgments}

This work was supported by a Grant-in-Aid for Specially Promoted Research (23000012, to CS) and a Grant-in-Aid for Scientific Research (C) (25460527 to HA). Part of this work was supported by grants from the Naito Foundation (HA) and Mochida Memorial Foundation for Medical and Pharmaceutical Research (HA).

LPS independent of TLR4. Science (2013) 341:1246-9. doi:10.1126/science. 1240248

15. Willingham SB, Bergstralh DT, O'Connor W, Morrison AC, Taxman DJ, Duncan JA, et al. Microbial pathogen-induced necrotic cell death mediated by the inflammasome components CIAS1/cryopyrin/NLRP3 and ASC. Cell Host Microbe (2007) 2:147-59. doi:10.1016/j.chom.2007.07.009

16. Miao EA, Mao DP, Yudkovsky N, Bonneau R, Lorang CG, Warren SE, et al. Innate immune detection of the type III secretion apparatus through the NLRC4 inflammasome. Proc Natl Acad Sci U S A (2010) 107:3076-80. doi:10.1073/pnas. 0913087107

17. Suzuki T, Franchi L, Toma C, Ashida H, Ogawa M, Yoshikawa Y, et al. Differential regulation of caspase-1 activation, pyroptosis, and autophagy via Ipaf and ASC in Shigella-infected macrophages. PLoS Pathog (2007) 3:e111. doi:10.1371/journal.ppat.0030111

18. Davis BK, Roberts RA, Huang MT, Willingham SB, Conti BJ, Brickey WJ, et al. Cutting edge: NLRC5-dependent activation of the inflammasome. J Immunol (2011) 186:1333-7. doi:10.4049/jimmunol.1003111

19. Rayamajhi M, Zak DE, Chavarria-Smith J, Vance RE, Miao EA. Cutting edge: mouse NAIP1 detects the type III secretion system needle protein. J Immunol (2013) 191:3986-9. doi:10.4049/jimmunol.1301549

20. Yang J, Zhao Y, Shi J, Shao F. Human NAIP and mouse NAIP1 recognize bacterial type III secretion needle protein for inflammasome activation. Proc Natl Acad Sci U S A (2013) 110:14408-13. doi:10.1073/pnas.1306376110

21. Suzuki S, Franchi L, He Y, Muñoz-Planillo R, Mimuro H, Suzuki T, et al. Shigella type III secretion protein MxiI is recognized by Naip2 to induce Nlrc4 inflammasome activation independently of Pkcס. PLoS Pathog (2014) 10:e1003926. doi:10.1371/journal.ppat.1003926

22. Lightfield KL, Persson J, Brubaker SW, Witte CE, von Moltke J, Dunipace EA, et al. Critical function for Naip5 in inflammasome activation by a conserved carboxy-terminal domain of flagellin. Nat Immunol (2008) 9:1171-8. doi:10. 1038/ni.1646

23. Kofoed EM, Vance RE. Innate immune recognition of bacterial ligands by NAIPs determines inflammasome specificity. Nature (2011) 477:592-5. doi:10. 1038/nature10394

24. Zhao Y, Yang J, Shi J, Gong YN, Lu Q, Xu H, et al. The NLRC4 inflammasome receptors for bacterial flagellin and type III secretion apparatus. Nature (2011) 477:596-600. doi:10.1038/nature10510

25. Tenthorey JL, Kofoed EM, Daugherty MD, Malik HS, Vance RE. Molecular basis for specific recognition of bacterial ligands by NAIP/NLRC4 inflammasomes. Mol Cell (2014) 54:17-29. doi:10.1016/j.molcel.2014.02.018

26. Senerovic L, Tsunoda SP, Goosmann C, Brinkmann V, Zychlinsky A, Meissner $\mathrm{F}$, et al. Spontaneous formation of IpaB ion channels in host cell membranes reveals how Shigella induces pyroptosis in macrophages. Cell Death Dis (2012) 3:e384. doi:10.1038/cddis.2012.124 
27. Miao EA, Leaf IA, Treuting PM, Mao DP, Dors M, Sarkar A, et al. Caspase-1induced pyroptosis is an innate immune effector mechanism against intracellular bacteria. Nat Immunol (2010) 11:1136-42. doi:10.1038/ni.1960

28. Suzuki S, Mimuro H, Kim M, Ogawa M, Ashida H, Toyotome T, et al. Shigella IpaH7.8 E3 ubiquitin ligase targets glomulin and activates inflammasomes to demolish macrophages. Proc Natl Acad Sci U S A (2014) 111:E4254-63. doi:10. 1073/pnas.1324021111

29. Rohde JR, Breitkreutz A, Chenal A, Sansonetti PJ, Parsot C. Type III secretion effectors of the IpaH family are E3 ubiquitin ligase. Cell Host Microbe (2007) 1:77-83. doi:10.1016/j.chom.2007.02.002

30. Ashida H, Kim M, Sasakawa C. Manipulation of the host cell death pathway by Shigella. Cell Microbiol (2014) 16:1757-66. doi:10.1111/cmi.12367

31. Carneiro LA, Travassos LH, Soares F, Tattoli I, Magalhaes JG, Bozza MT, et al. Shigella induces mitochondrial dysfunction and cell death in nonmyleoid cells. Cell Host Microbe (2009) 5:123-36. doi:10.1016/j.chom.2008.12.011

32. Niebuhr K, Giuriato S, Pedron T, Philpott DJ, Gaits F, Sable J, et al. Conversion of PtdIns $(4,5) \mathrm{P}(2)$ into PtdIns(5)P by the $S$. flexneri effector IpgD reorganizes host cell morphology. EMBO J (2002) 21:5069-78. doi:10.1093/ emboj/cdf522

33. Pendaries C, Tronchère H, Arbibe L, Mounier J, Gozani O, Cantley L, et al. PtdIns5P activates the host cell PI3-kinase/Akt pathway during Shigella flexneri infection. EMBO J (2006) 25:1024-34. doi:10.1038/sj.emboj.7601001

34. Ramel D, Lagarrigue F, Pons V, Mounier J, Dupuis-Coronas S, Chicanne G, et al. Shigella flexneri infection generates the lipid PI5P to alter endocytosis and prevent termination of EGFR signaling. Sci Signal (2011) 4:ra61. doi:10.1126/ scisignal.2001619

35. Bergounioux J, Elisee R, Prunier AL, Donnadieu F, Sperandio B, Sansonetti P, et al. Calpain activation by the Shigella flexneri effector VirA regulates key steps in the formation and life of the bacterium's epithelial niche. Cell Host Microbe (2012) 11:240-52. doi:10.1016/j.chom.2012.01.013

36. Andree M, Seeger JM, Schüll S, Coutelle O, Wagner-Stippich D, Wiegmann K, et al. BID-dependent release of mitochondrial SMAC dampens XIAP-mediated immunity against Shigella. EMBO J (2014) 33:2171-87. doi:10.15252/embj. 201387244

37. Kobayashi T, Ogawa M, Sanada T, Mimuro H, Kim M, Ashida H, et al. The Shigella OspC3 effector inhibits caspase-4, antagonizes inflammatory cell death, and promotes epithelial infection. Cell Host Microbe (2013) 13:570-83. doi:10. 1016/j.chom.2013.04.012

38. Knodler LA, Crowley SM, Sham HP, Yang H, Wrande M, Ma C, et al. Noncanonical inflammasome activation of caspase-4/caspase-11 mediates epithelial defenses against enteric bacterial pathogens. Cell Host Microbe (2014) 16:249-56. doi:10.1016/j.chom.2014.07.002

39. Shi J, Zhao Y, Wang Y, Gao W, Ding J, Li P, et al. Inflammatory caspases are innate immune receptors for intracellular LPS. Nature (2014) 514:187-92. doi:10.1038/nature 13683

40. Travassos LH, Carneiro LA, Ramjeet M, Hussey S, Kim YG, Magalhães JG, et al. Nod1 and Nod2 direct autophagy by recruiting ATG16L1 to the plasma membrane at the site of bacterial entry. Nat Immunol (2010) 11:55-62. doi:10. 1038/ni.1823

41. Thurston TL, Wandel MP, von Muhlinen N, Foeglein A, Randow F. Galectin 8 targets damaged vesicles for autophagy to defend cells against bacterial invasion. Nature (2012) 482:414-8. doi:10.1038/nature10744

42. Dupont N, Lacas-Gervais S, Bertout J, Paz I, Freche B, Van Nhieu GT, et al. Shigella phagocytic vacuolar membrane remnants participate in the cellular response to pathogen invasion and are regulated by autophagy. Cell Host Microbe (2009) 6:137-49. doi:10.1016/j.chom.2009.07.005

43. Leung Y, Ally S, Goldberg MB. Bacterial actin assembly requires toca-1 to relieve N-wasp autoinhibition. Cell Host Microbe (2008) 3:39-47. doi:10.1016/j. chom.2007.10.011

44. Baxt LA, Goldberg MB. Host and bacterial proteins that repress recruitment of LC3 to Shigella early during infection. PLoS One (2014) 9:e94653. doi:10.1371/ journal.pone.0094653

45. Ogawa M, Yoshimori T, Suzuki T, Sagara H, Mizushima N, Sasakawa C. Escape of intracellular Shigella from autophagy. Science (2005) 307:727-31. doi:10. 1126/science.1106036

46. Kayath CA, Hussey S, El Hajjami N, Nagra K, Philpott D, Allaoui A. Escape of intracellular Shigella from autophagy requires binding to cholesterol through the type III effector, IcsB. Microbes Infect (2010) 12:956-66. doi:10.1016/j. micinf.2010.06.006
47. Huett A, Heath RJ, Begun J, Sassi SO, Baxt LA, Vyas JM, et al. The LRR and RING domain protein LRSAM1 is an E3 ligase crucial for ubiquitin-dependent autophagy of intracellular Salmonella typhimurium. Cell Host Microbe (2012) 12:778-90. doi:10.1016/j.chom.2012.10.019

48. Mostowy S, Bonazzi M, Hamon MA, Tham TN, Mallet A, Lelek M, et al. Entrapment of intracytosolic bacteria by septin cage-like structures. Cell Host Microbe (2010) 8:433-44. doi:10.1016/j.chom.2010.10.009

49. Mostowy S, Sancho-Shimizu V, Hamon MA, Simeone R, Brosch R, Johansen T, et al. p62 and NDP52 proteins target intracytosolic Shigella and Listeria to different autophagy pathways. J Biol Chem (2011) 286:26987-95. doi:10.1074/ jbc.M111.223610

50. McGhie EJ, Brawn LC, Hume PJ, Humphreys D, Koronakis V. Salmonella takes control: effector-driven manipulation of the host. Curr Opin Microbiol (2009) 12:117-24. doi:10.1016/j.mib.2008.12.001

51. Sherwood RK, Roy CR. A Rab-centric perspective of bacterial pathogenoccupied vacuoles. Cell Host Microbe (2013) 14:256-68. doi:10.1016/j.chom. 2013.08.010

52. Stenmark H. Rab GTPases as coordinators of vesicle traffic. Nat Rev Mol Cell Biol (2009) 10:513-25. doi:10.1038/nrm2728

53. Mellouk N, Weiner A, Aulner N, Schmitt C, Elbaum M, Shorte SL, et al. Shigella subverts the host recycling compartment to rupture its vacuole. Cell Host Microbe (2014) 16:517-30. doi:10.1016/j.chom.2014.09.005

54. Dong N, Zhu Y, Lu Q, Hu L, Zheng Y, Shao F. Structurally distinct bacterial TBC-like GAPs link Arf GTPase to Rab1 inactivation to counteract host defenses. Cell (2012) 150:1029-41. doi:10.1016/j.cell.2012.06.050

55. Burnaevskiy N, Fox TG, Plymire DA, Ertelt JM, Weigele BA, Selyunin AS, et al. Proteolytic elimination of $N$-myristoyl modifications by the Shigella virulence factor IpaJ. Nature (2013) 496:106-9. doi:10.1038/nature12004

56. Burnaevskiy N, Peng T, Reddick LE, Hang HC, Alto NM. Myristoylome profiling reveals a concerted mechanism of ARF GTPase deacylation by the bacterial protease IpaJ. Mol Cell (2015) 58:110-22. doi:10.1016/j.molcel.2015.01.040

57. Mounier J, Boncompain G, Senerovic L, Lagache T, Chrétien F, Perez F, et al. Shigella effector IpaB-induced cholesterol relocation disrupts the Golgi complex and recycling network to inhibit host cell secretion. Cell Host Microbe (2012) 12:381-9. doi:10.1016/j.chom.2012.07.010

58. Puhar A, Tronchère H, Payrastre B, Nhieu GT, Sansonetti PJ. A Shigella effector dampens inflammation by regulating epithelial release of danger signal ATP through production of the lipid mediator PtdIns5P. Immunity (2013) 39:1121-31. doi:10.1016/j.immuni.2013.11.013

59 . Hayden MS, Ghosh S. NF- $\kappa B$, the first quarter-century: remarkable progress and outstanding questions. Genes Dev (2012) 26:203-34. doi:10.1101/gad. 183434.111

60. Ashida H, Kim M, Sasakawa C. Exploitation of the host ubiquitin system by human bacterial pathogens. Nat Rev Microbiol (2014) 12:399-413. doi:10.1038/ nrmicro3259

61. Sanada T, Kim M, Mimuro H, Suzuki M, Ogawa M, Oyama A, et al. The Shigella flexneri effector OspI deamidates UBC13 to dampen the inflammatory response. Nature (2012) 483:623-6. doi:10.1038/nature10894

62. Ashida H, Nakano H, Sasakawa C. Shigella IpaH0722 E3 ubiquitin ligase effector targets TRAF2 to inhibit PKC-NF- $\kappa$ B activity in invaded epithelial cells. PLoS Pathog (2013) 9:e1003409. doi:10.1371/journal.ppat.1003409

63. Ashida H, Kim M, Schmidt-Supprian M, Ma A, Ogawa M, Sasakawa C. A bacterial E3 ubiquitin ligase IpaH9.8 targets NEMO/IKK $\gamma$ to dampen the host NF- $\mathrm{BB}$-mediated inflammatory response. Nat Cell Biol (2010) 12:66-73. doi:10. 1038/ncb2006

64. Kim DW, Lenzen G, Page AL, Legrain P, Sansonetti PJ, Parsot C. The Shigella flexneri effector OspG interferes with innate immune responses by targeting ubiquitin-conjugating enzymes. Proc Natl Acad Sci U S A (2005) 102:14046-51. doi:10.1073/pnas.0504466102

65. Zhou Y, Dong N, Hu L, Shao F. The Shigella type three secretion system effector OspG directly and specifically binds to host ubiquitin for activation. PLoS One (2013) 8:e57558. doi:10.1371/journal.pone.0057558

66. Pruneda JN, Smith FD, Daurie A, Swaney DL, Villén J, Scott JD, et al. E2 Ub conjugates regulate the kinase activity of Shigella effector OspG during pathogenesis. EMBO J (2014) 33:437-49. doi:10.1002/embj.201386386

67. Grishin AM, Condos TE, Barber KR, Campbell-Valois FX, Parsot C, Shaw GS, et al. Structural basis for the inhibition of host protein ubiquitination by Shigella effector kinase OspG. Structure (2014) 22:878-88. doi:10.1016/j.str. 2014.04.010 
68. Arbibe L, Kim DW, Batsche E, Pedron T, Mateescu B, Muchardt C, et al. An injected bacterial effector targets chromatin access for transcription factor NF$\kappa \mathrm{B}$ to alter transcription of host genes involved in immune responses. Nat Immunol (2007) 8:47-56. doi:10.1038/ni1423

69. Li H, Xu H, Zhou Y, Zhang J, Long C, Li S, et al. The phosphothreonine lyase activity of a bacterial type III effector family. Science (2007) 315:1000-3. doi:10.1126/science. 1138960

70. Harouz H, Rachez C, Meijer BM, Marteyn B, Donnadieu F, Cammas F, et al. Shigella flexneri targets the HP1 $\gamma$ subcode through the phosphothreonine lyase OspF. EMBO J (2014) 33:2606-22. doi:10.15252/embj.201489244

71. Okuda J, Toyotome T, Kataoka N, Ohno M, Abe H, Shimura Y, et al. Shigella effector IpaH9.8 binds to a splicing factor U2AF(35) to modulate host immune responses. Biochem Biophys Res Commun (2005) 333:531-9. doi:10.1016/j.bbrc. 2005.05.145

72. Le-Barillec K, Magalhaes JG, Corcuff E, Thuizat A, Sansonetti PJ, Phalipon A, et al. Roles for T and NK cells in the innate immune response to Shigella flexneri. J Immunol (2005) 175:1735-40. doi:10.4049/jimmunol.175.3.1735

73. Sellge G, Magalhaes JG, Konradt C, Fritz JH, Salgado-Pabon W, Eberl G, et al. Th17 cells are the dominant $T$ cell subtype primed by Shigella flexneri mediating protective immunity. J Immunol (2010) 184:2076-85. doi:10.4049/jimmunol. 0900978

74. Jehl SP, Doling AM, Giddings KS, Phalipon A, Sansonetti PJ, Goldberg MB, et al. Antigen-specific CD8(+) T cells fail to respond to Shigella flexneri. Infect Immun (2011) 79:2021-30. doi:10.1128/IAI.00939-10

75. Konradt C, Frigimelica E, Nothelfer K, Puhar A, Salgado-Pabon W, di Bartolo $\mathrm{V}$, et al. The Shigella flexneri type three secretion system effector IpgD inhibits
T cell migration by manipulating host phosphoinositide metabolism. Cell Host Microbe (2011) 9:263-72. doi:10.1016/j.chom.2011.03.010

76. Salgado-Pabón W, Celli S, Arena ET, Nothelfer K, Roux P, Sellge G, et al. Shigella impairs T lymphocyte dynamics in vivo. Proc Natl Acad Sci U S A (2013) 110:4458-63. doi:10.1073/pnas.1300981110

77. Raqib R, Ekberg C, Sharkar P, Bardhan PK, Zychlinsky A, Sansonetti PJ, et al. Apoptosis in acute shigellosis is associated with increased production of Fas/Fas ligand, perforin, caspase-1, and caspase- 3 but reduced production of Bcl-2 and interleukin-2. Infect Immun (2002) 70:3199-207. doi:10.1128/IAI.70. 6.3199-3207.2002

78. Nothelfer K, Arena ET, Pinaud L, Neunlist M, Mozeleski B, Belotserkovsky I, et al. B lymphocytes undergo TLR2-dependent apoptosis upon Shigella infection. J Exp Med (2014) 211:1215-29. doi:10.1084/jem.20130914

Conflict of Interest Statement: The authors declare that the research was conducted in the absence of any commercial or financial relationships that could be construed as a potential conflict of interest.

Copyright (c) 2015 Ashida, Mimuro and Sasakawa. This is an open-access article distributed under the terms of the Creative Commons Attribution License (CC BY). The use, distribution or reproduction in other forums is permitted, provided the original author(s) or licensor are credited and that the original publication in this journal is cited, in accordance with accepted academic practice. No use, distribution or reproduction is permitted which does not comply with these terms. 